\title{
Factores psicosexuales y habilidades sociosexuales en los adultos mayores: una revisión sistemática de la literatura.
}

DOI: $10.46932 / \mathrm{sfjdv2n3-003}$

Received in: May 1st, 2021

Accepted in: Jun 30th, 2021

\author{
Xavier Fabricio Reyes Trelles \\ Psicólogo Clínico \\ Ministerio de Inclusión Económica y Social \\ Ecuador, Cañar, Azogues \\ E-mail: fabricioreyestrelles@gmail.com
}

\section{RESUMEN}

La particularidad actual de varios países es el envejecimiento poblacional, la cual visibiliza a los adultos mayores (AM) como individuos con propias necesidades y permite cuestionar las condiciones en las que están envejecimiento, en especial, en el ámbito sexual expresado por los factores psicosexuales y las habilidades sociosexuales, mismo que denota vacíos investigativos. El objetivo de la investigación es identificar y sintetizar la información disponible sobre los factores psicosexuales y habilidades sociosexuales en un segmento específico de AM. Se realizó una revisión de la literatura científica en la última década por medio de 4 bases de datos digitales Scopus, Scielo, Dialnet y Web of Science, posterior a la lectura y análisis a texto completo se seleccionaron estudios cuantitativos, cualitativos y revisiones de la literatura asegurando su aporte de interés por medio de los criterios de selección. Como resultados, la revisión arrojó 42 referencias utilizadas para sintetizar la información disponible, posterior al análisis, los datos fueron agrupados en factores psicosexuales, expresados en 6 variables, habilidades sociosexuales, expresados en 3 variables, y la edad, entendida como una variable transversal a todas las mencionadas. Concluyendo, esta revisión de la literatura aporta una síntesis integral de las variables que intervienen en la sexualidad de los AM, que a su vez permitirá comprenderlas y canalizarlas por su impacto directo en el proceso de envejecimiento. Finalmente, facilitará la construcción teórica de la temática que guíen la estructuración de políticas públicas socio sanitarias al momento de desarrollar intervenciones exitosas de salud.

Palabras clave: factores psicosexuales, habilidades sociosexuales, sexualidad, satisfacción sexual, funcionamiento sexual, adultos mayores.

\begin{abstract}
The current particularity of several countries is the aging population, which makes older adults (OA) visible as individuals with their own needs and allows questioning the conditions in which they are aging, especially in the sexual sphere expressed by psychosexual factors and sociosexual skills, which denotes research gaps. The objective of the research is to identify and synthesize the available information on psychosexual factors and sociosexual skills in a specific segment of OA. A review of the scientific literature in the last decade was conducted through 4 digital databases Scopus, Scielo, Dialnet and Web of Science, after the reading and analysis of the full text, quantitative, qualitative studies and literature reviews were selected, ensuring their contribution of interest by means of the following selection criteria. As results, the review yielded 42 references used to synthesize the available information, after analysis, the data were grouped into psychosexual factors, expressed in 6 variables, sociosexual skills, expressed in 3 variables, and age, understood as a cross-cutting variable to all those mentioned. As a conclusion, this literature review provides a comprehensive synthesis of the variables involved in the sexuality of OA, which in turn will allow understanding and channeling them for their direct impact on the aging process.
\end{abstract}


Finally, it will facilitate the theoretical construction of the thematic that will guide the structuring of public socio health policies at the time of developing successful health interventions.

Key words: psychosexual factors, sociosexual skills, sexuality, sexual satisfaction, sexual functioning, older adults.

\section{INTRODUCCIÓN}

Los individuos a partir de los 60 años de edad son considerados como adultos mayores (AM) (Organizacion Mundial de la Salud [OMS], 2015). Constituyen el segmento de más rápido crecimiento en todo el mundo y se espera que continúe así a lo largo del siglo (Morris \& Maisto, 2009), llegando a duplicarse para 2050, esto es del 11\% al 22\% de la población mundial (OMS, 2015). Este aumento significativo los visibiliza socialmente con necesidades propias en todas sus áreas. La sexualidad de los AM ha sido descuidada puesto que la sociedad prioriza las características juveniles, existen un gran número de estereotipos interiorizados culturalmente (Llanes, 2013) y el escaso interés al momento de elaborar políticas públicas, particularmente en Ecuador (Ministerio de Inclusión Económica y Social, 2018).

La teoría integrativa de la sexualidad concibe que el ser humano es sexuado durante toda su existencia (Rubio, 2014), con variaciones conforme el envejecimiento (Llanes, 2013; Sierra, et al., 2014). La sexualidad humana, entendida como una construcción sociocultural (Rubio, 2014), responde a un proceso neurológico, psicológico, endocrino, y fisiológico que repercute directamente en la salud integral (SI) y calidad de vida (CV) de los AM (López et al., 2019; Sierra et al., 2014; Srinivasan et al., 2019). En los AM, la frecuencia de las expresiones sexuales disminuyen sin desaparecen (DeLamater, 2012; Torres \& Rodríguez, 2019) rescatando otras conductas como besos y caricias (Llanes, 2013).

El estudio de la sexualidad en la adultez mayor apenas ha iniciado desde comienzos del presente siglo de forma descriptiva de las conductas y los cambios fisiológicos (DeLamater, 2012), encontrando su punto álgido en las investigaciones de la respuesta sexual de Masters y Johnson (Morris \& Maisto, 2009). Con mayor actualidad, se evidencian estudios respecto a los comportamientos sexuales inapropiados en la demencia desde un enfoque farmacológico (Srinivasan et al., 2019). No obstante, la apreciación de otras variables de índole psico y socio sexual no han tenido el mismo desenlace (DeLamater, 2012), pese a que los diversos aspectos emocionales, cognitivos, culturales, éticos y sociales determinan la sexualidad (Sierra et al., 2014).

Los factores psicosexuales se expresan en actitud hacia la sexualidad, actitud frente a los estímulos y fantasías sexuales, estereotipos y prejuicios, satisfacción sexual, autoestima, funcionamiento sexual y otras variables psicológicas. Al mismo tiempo, las habilidades sociosexuales se expresan en conducta 
sexual, asertividad sexual y manejo de información. Los mencionados se entrelazan, son interdependientes y en su totalidad se proponen como predictores de la sexualidad en los AM (DeLamater, 2012; Sierra et al., 2014).

Estudios como el de Sierra et al. (2014) evidenciaron una correlación significativa entre los factores psicosexuales y el funcionamiento sexual. Llanes (2013) propone que los marcados prejuicios sociales sobre la sexualidad en la adultez mayor conllevan un empobrecimiento emocional y disminución en la percepción de la CV. Por último, López et al. (2019) resaltan que la satisfacción sexual y el mantenimiento de la actividad sexual repercute directamente en la SI y CV de los AM.

Después de mencionar los vacíos investigativos de los factores determinantes de la sexualidad, el aumento exponencial de este grupo etario desconociendo las condiciones de salud sexual en las que envejecen, la escasa atención prestada por las políticas públicas y el impacto que tienen sobre la SI y CV. El objetivo es identificar y sintetizar la información disponible sobre los factores psicosexuales y habilidades sociosexuales en un segmento específico de AM, mismo que, podría ser la base para proponer planes integrales de intervención acorde a las necesidades sexuales específicas de los AM.

\section{METODOLOGÍA}

Para la revisión sistemática se siguieron los lineamientos de la Presentación Referida de Informes de Revisión Sistemática y Meta-Análisis (PRISMA) y la lista de verificación de los elementos para las revisiones (Moher et al., 2009). Se realizó una búsqueda estructurada en 4 bases de datos electrónicas, Scopus, Scielo, Dialnet y Web of Science. Como criterios de búsqueda se utilizaron los términos claves en castellano e inglés: Sexualidad/Sexuality, Comportamiento sexual/Sexual behavior, Satisfacción sexual/Sexual satisfaction, Asertividad sexual/Sexual assertiveness y Funcionamiento sexual/Sexual functioning en combinación con el término Adulto mayor/Older people correspondientemente.

Para la selección de artículos se emplearon los siguientes criterios inclusión: a) estudios cuantitativos, cualitativos y revisiones bibliográficas que indagaron sobre factores psicosexuales y habilidades sociosexuales; b) investigaciones en población no institucionalizadas; c) estudios en personas mayores de 60 años (exceptuando dos investigaciones por sus datos de interés), y; d) publicaciones desde 2010 hasta 2020 en castellano o inglés. Criterios de exclusión: a) investigaciones que analizaran la perspectiva de familiares o personal sanitario; b) estudio de caso o análisis de los resultados de una intervención, y; c) investigaciones que consideraron, 
exclusivamente, población LGBTQ, con disfuncionalidad sexual y/o con un trastorno psicológico diagnosticado. Además, con los criterios se buscó homogenizar el segmento poblacional.

La búsqueda de información y selección de artículos siguió el diagrama de flujo PRISMA y se encuentra ilustrado en la figura 1. Además, la selección de artículos tuvo en cuenta referencias que aseguraran el aporte de interés, confiabilidad y validez. Finalmente, 42 artículos conforman esta revisión que fueron sometidos a un protocolo de extracción de datos basado en fichas de lectura, mismos que, permitió sintetizar la información relevante en concordancia con las variables mencionadas y presentadas en el apartado de resultados.

Figura 1. Diagrama de flujo PRISMA

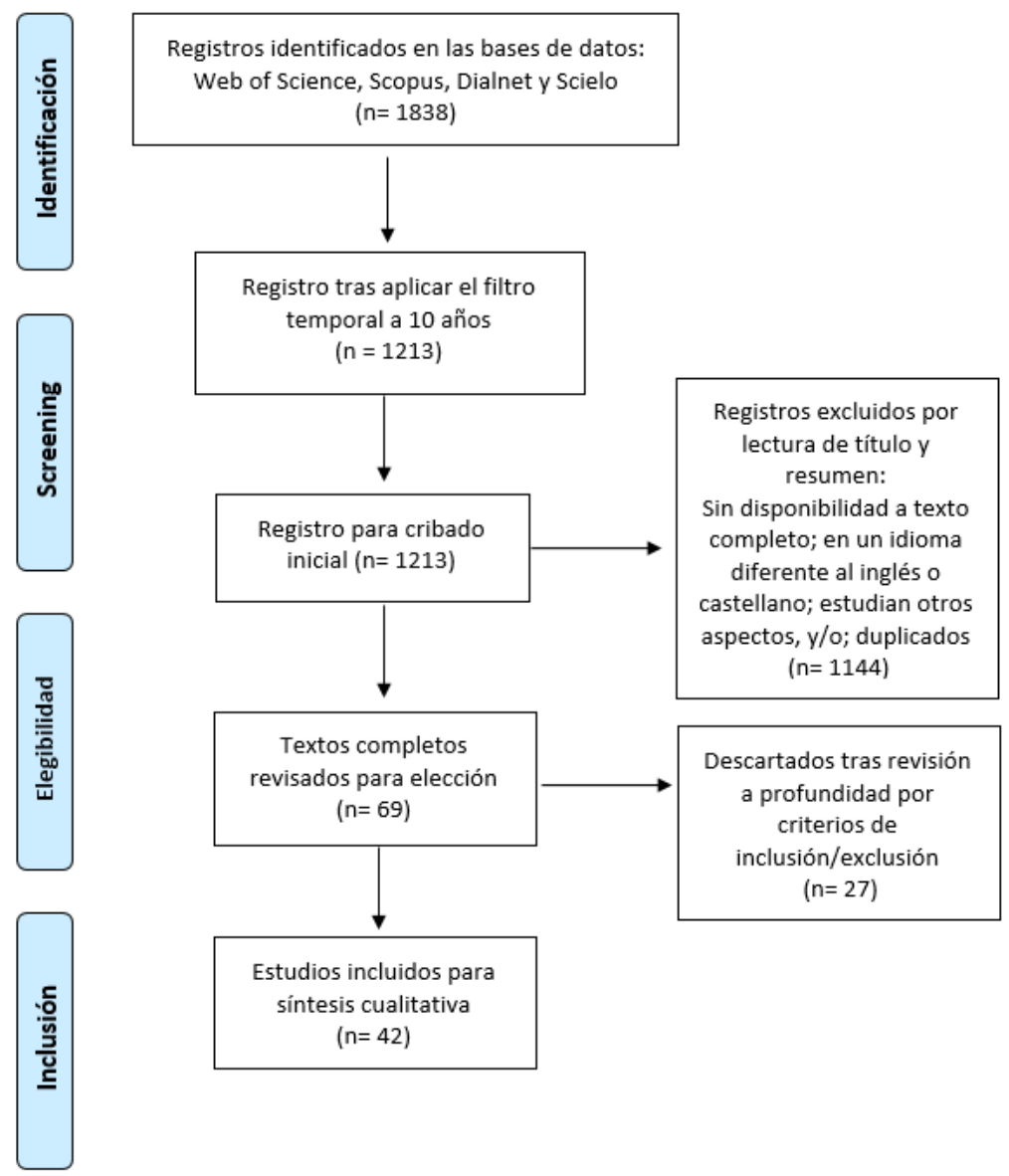

Nota: Modelo gráfico de Moher et al., (2009) en Preferred Reporting Items for Systematic Reviews and Meta-Analyses: The PRISMA Statement.

\section{RESULTADOS}

Como consideraciones iniciales respecto al estudio de la sexualidad, no hay un consenso científico sobre la terminología (DeLamater, 2012) y la forma de medir las variables (Can et al., 2016). Generalmente, los estudios han sido enfocados en población heterosexual (Sinković \& Towler, 2019), con pareja (Perdomo et al., 2013) y en menores de 70 años (Hsu et al., 2018). Los hombres tienden a participar 
Tabla 2. Esquematización y generalidades metodológicas

\begin{tabular}{ll}
\hline Metodología & Autor/es, año (muestra en número de participantes) \\
\hline Estudios cuantitativos- & Olivet et al., 2014 (111); Monteagudo et al., 2016 (510*); \\
descriptivos & Jalenques et al., $2020(184 * *) ;$ Traeen et al., $2018\left(3816^{* * * *)} ;\right.$ \\
& Cybulski et al., 2018 (170); Robledo et al., $2020(665) ;$ Perdomo \\
& et al., 2013 (200); Díaz et al., 2015 (90); Uchôa et al., 2016 (90); \\
& Hernández et al., 2019 (138); Linares et al., 2018 (147); Castro et \\
& al., 2017 (423***); Cabrera et al., 2018 (65); Yang y Yan, 2015 \\
& (688); Heywood et al., 2017 (1583)
\end{tabular}

Estudios cuantitativos- Sierra et al., 2014 (405); Morell et al., 2018 (70); Flynn y Gow, correlacionales 2015 (134)

Estudios longitudinales Trudel et al., 2014 (394 parejas) a 18 meses; Hsu et al., 2018 (986) a 2 y 5 años

Estudios comparativos Beckman et al., 2014 en 4 cohortes a 30 años

Estudios cualitativos Gharibi et al., 2018 (27**); Can et al., 2015 (50); Da Silva et al., 2019 (19**); Iacub et al., 2020 (40); Fileborn et al., 2017 (53); Ménard et al., 2015 (30); Hurd y Mahal, 2019 (22); Ševčíková y Sedláková, 2020

\begin{tabular}{ll}
\hline Metodología & Autor/es, año (selección final de artículos) \\
\hline Revisiones sistemáticas & $\begin{array}{l}\text { Torres y Rodríguez, } 2019 \text { (11); Sinković y Towler, } 2018 \text { (69); } \\
\text { Gewirtz et al., } 2018 \text { (20); Hartmans et al., 2014 (8) }\end{array}$
\end{tabular}

Revisiones y síntesis de Llanes, 2013; DeLamater, 2012; Trudel et al., 2010; Slack y Aziz, la literatura

Fuente: elaboración propia

Nota: *Exclusivamente hombres **Exclusivamente mujeres ***Exclusivamente población mayor de 75 años *****Exclusivamente población menor de 75 años

\subsection{FACTORES PSICOSEXUALES}

La actitud hacia la sexualidad es definida a manera de valoración positiva o negativa sobre un objeto/situación específica, relativamente estable y resistente al cambio (Traeen et al., 2018). Se deriva de experiencias individuales y valores globales establecidos socialmente como la religión y la moralidad (Traeen et al., 2018). Las actitudes sexuales devienen de guiones sexuales instaurados culturalmente que estructuran la percepción de la realidad (Olivet et al., 2014; Traeen et al., 2018). Por lo tanto, óptimas actitudes se consiguen con el manejo efectivo de los mensajes sociales disruptivos sobre el sexo (Ménard et al., 2015), caso contrario, afectan y son capaces de suprimir el deseo sexual al ser considerado inapropiados (Cabrera et al., 2018; Morell et al., 2018). 
Se evidenció predominancia del estudio de esta variable. Mayoritariamente los AM consideran que los hombres están más interesados por el sexo (Gewirtz et al., 2019) y menor edad de los AM se relaciona con mayor facilidad para de sexualidad (Cabrera et al., 2018; Sinković \& Towler, 2019). Por su parte, las mujeres con mayor frecuencia inhiben sus deseos al tener normas sociales más restrictivas (Ševčíková \& Sedláková, 2020) y las viudas apegadas a la religión, ligan la sexualidad con la reproducción y desmerecen el factor placer (Torres \& Rodríguez, 2019).

En relación al emplazamiento, una investigación mexicana refiere que el 59\% de la muestra estudiada no consideró perjudicial mantener relaciones sexuales en la vejez (Can et al., 2016). Asimismo, el $72 \%$ de AM cubanos manifestaron que es una fuente de placer, bueno y saludable, y debe ceder conforme envejecien por ser innecesario/inadecuado (Díaz et al., 2015). Otro estudio cubano encontró que el $80 \%$ de los hombres lo consideraron una práctica beneficiosa para la salud, en contraposición de lo manifestado por el 60\% de las mujeres (Perdomo et al., 2013). Por su parte, mujeres brasileñas exhibieron actitudes favorables y aseguraron que su expresión verbal depende de los sentimientos que genera y el contexto (Da Silva et al., 2019).

En Europa, un estudio determina que los portugueses consideraron positivamente al sexo en relación al bienestar integral, siendo el envejecimiento un obstáculo en el disfrute sexual para los hombres, en contraste a lo referido por las mujeres portuguesas; los hombres noruegos y mujeres danesas no consideraron al envejecimiento como un impedimento para mantener relaciones sexuales, no obstante, refirieron que no es beneficioso; los hombres belgas y daneses manifestaron que el sexo no es importante y sus cambios son consecuencia del envejecimiento (Traeen et al., 2018). Otros estudios evidenciaron que, los polacos tienen actitudes moderadas hacia la sexualidad (Cybulski et al., 2018) y el 53\% de AM españoles consideraron la importancia de la sexualidad (Hernández et al., 2019). En China, Yang \& Yan (2016) afirman que la mitad de la población de AM mantienen una actitud de interés hacia lo sexual.

DeLamater (2012), Kleinstäuber (2017) y Beckman et al., (2014) proponen que la actitud hacia lo sexual tiene relación directamente proporcional con el interés sexual, la frecuencia de la actividad (Traeen et al., 2018), el deseo (Trudel et al., 2014), la expresión sexual (Da Silva et al., 2019; Torres \& Rodríguez, 2019), la satisfacción sexual (Cybulski et al., 2018) y menor percepción del envejecimiento (Da Silva et al., 2019). Por su parte, las actitudes hacia la sexualidad repercuten notoriamente en la CV y bienestar integral de los AM, en especial, cuando tienen parejas estables (DeLamater, 2012). Actitudes sexuales liberales y adecuado bienestar psicológico se relacionan con vivir en comunidad (Torres \& Rodríguez, 2019) y mantener una pareja estable (Morell et al., 2018). Finalmente, actitud sexual positiva y adecuada expresión sexual son decisivas para mantener actividad sexual de calidad (Perdomo et al., 2013). 
Respecto con la actitud hacía los estímulos y fantasías sexuales, la literatura es limitada. Ambas variables, destacándose el fantaseo, excitan y potencian el deseo sexual (Iacub et al., 2020). Destaca que actitudes negativas y descensos en las fantasías se relacionan con menores niveles de funcionamiento sexual (Trudel et al., 2010), especialmente en mujeres (Sierra et al., 2014). Consecuentemente, Sierra et al. (2014) afirman que las actitudes positivas hacia los estímulos y fantasías conjuntamente con respuestas asertivas predicen significativamente el adecuado funcionamiento sexual. Además, apertura a las experiencias, poner atención y enfocarse en las prácticas sexuales les ayuda a mantenerse activos sexualmente (Ménard et al., 2015).

La principal barrera en su expresión es la escasa privacidad e intimidad (Fileborn et al., 2017; Torres \& Rodríguez, 2019), esencialmente, por la convivencia y/o cuidar familiares (Gharibi et al., 2019). Ejemplificando, en tres estudios cubanos los AM refirieron falta de privacidad en los siguientes porcentajes el 68\%, (Díaz et al., 2015) el 76\% (Perdomo et al., 2013) y 90\% (Linares et al., 2018).

Únicamente, Yang y Yan (2016) encontró que la población china expresó actitud positiva al uso de ayudas técnicas sexuales. En relación con la pornografía, Castro et al., (2018) describen que de los AM españoles con acceso a internet el $69 \%$ lo ha utilizado con fines sexuales y con una frecuencia semanal de 3 horas en hombres y de 30 minutos en mujeres, el $88 \%$ vio imágenes o películas pornográficas y el 55\% lo usó con el fin de distraerse.

Por lo que se refiere a los estereotipos y prejuicios, entendidos como imágenes mentales sobre un grupo y valoraciones a priori respectivamente, son variables de carácter social que al ser interiorizadas dictan el comportamiento (Llanes, 2013) y la expresión sexual (Gharibi et al., 2019) de los AM. La religión con sus mandatos, la familia con su opresión y el personal de salud con su falta de educación ayudan a configurar los prejuicios inhibidores de la expresión sexual (Jalenques et al., 2020; Perdomo et al., 2013; Ramírez et al., 2017; Ševčíková \& Sedláková, 2020; Uchôa et al., 2016). Además, Ezhova et al. (2020) afirman que ciertos constructos culturales vinculan la sexualidad con la inmoralidad, situación ahondada por factores de género, raza y edad.

Los prejuicios más detectados en la adultez mayor hacen referencia a la asexualidad (Fileborn et al., 2017; Llanes, 2013; Torres \& Rodríguez, 2019), incapacidad de producir placer erótico por sus cambios estéticos (Iacub et al., 2020; Llanes, 2013), asociación exclusiva entre sexo y reproducción (Llanes, 2013; Torres \& Rodríguez, 2019) y el viagra como fomentador del sexo desenfrenado (Sinković \& Towler, 2019). Un prejuicio en favor de la actividad sexual es la sensación del deber marital (Gharibi et al., 2019; Torres \& Rodríguez, 2019). 
Siguiendo la misma línea, Gewirtz et al. (2019) resaltan el conflicto en los AM entre el deseo de experimentar la sexualidad contra las concepciones sociales preestablecidas. Can et al. (2016) afirman que, principalmente las mujeres, consideraron más importante la opinión social que seguir sus deseos y emociones. Igualmente, Morell et al. (2018) afirman que los AM con intenso deseo sexual vivencian sentimientos de culpa y vergüenza a causa de los prejuicios. Para concluir, los AM refirieron que el personal sanitario invisibiliza sus deseos al tener una pobre educación sexual (Ševčíková \& Sedláková, 2020) y se sienten rechazados (Torres \& Rodríguez, 2019).

Por otra parte, la satisfacción sexual es una sensación subjetiva de bienestar que engloba expectativas, emociones y afectos producto de satisfacer necesidades, deseos y pasión sexual (Olivet et al., 2014; Ramírez et al., 2017). Se logra sin la necesidad de culminar en una situación meramente genital (Ramírez et al., 2017), aunque exista hegemonía de este aspecto al investigar la temática (Gewirtz et al., 2019). Sin embargo, apenas un tercio de AM hombres cubanos consideró la penetración como necesaria para lograr satisfacción (Can et al., 2016), misma situación mencionada por la mitad de AM españoles (Cabrera et al., 2018).

Varios países muestran proporciones considerables de satisfacción sexual en sus AM (Traeen et al., 2017). Estudios cubanos, señalan satisfacción en el 58\% de la población (Olivet et al., 2014; Perdomo et al., 2013), enfatizando que, Monteagudo et al. (2016) hallan que el 78\% de hombres reportó satisfacción con su deseo sexual pese a disminuir y Linares et al. (2018) aseveran que el $92 \%$ de hombres y el $88 \%$ de mujeres activos sexualmente manifestaron satisfacción, mientras que, el $88 \%$ de hombres y $65 \%$ de mujeres inactivos reportaron insatisfacción. En Polonia, los AM atestiguaron niveles medianos de satisfacción (Cybulski et al., 2018) y en Australia, el 79\% dijo estar al menos medianamente satisfecho sexualmente (Heywood et al., 2017).

Los AM con menos de una relación sexual al mes y quienes desean incrementar su frecuencia exteriorizan menor satisfacción sexual (Heywood et al., 2017). A su vez, la satisfacción sexual repercute en la relación marital, la frecuencia de los encuentros sexuales (Trudel et al., 2010), el placer reportado (Monteagudo et al., 2016) e influye en el desarrollo de la autoestima y las emociones (Ramírez et al., 2017). Finalmente, existe influye directamente en la adecuada percepción de CV (Heywood et al., 2017; Ramírez et al., 2017) y buen estado de SI (López et al., 2019), especialmente respecto a la salud mental en mujeres (Heywood et al., 2017).

Por lo que se refiere a otras variables psicológicas, fueron detectados ciertos factores emocionales, relacionales y cognitivos que se desglosan a continuación:

Emociones consideradas negativas, como tristeza, miedo e ira, se relacionan con esconder impulsos sexuales (Torres \& Rodríguez, 2019) y disminuir la frecuencia de los encuentros sexuales 
(Kleinstäuber, 2017; Sinković \& Towler, 2019). La depresión, ansiedad e irritabilidad y el uso de psicotrópicos para trastornos emocionales se asocian con alteraciones de la sexualidad de los AM (Gharibi et al., 2019; López et al., 2019; Morell et al., 2018; Slack \& Aziz, 2020; Torres \& Rodríguez, 2019). También, las investigaciones acreditan otros factores emocionales que repercuten en la sexualidad sin establecer su magnitud, estos son: vergüenza (Da Silva et al., 2019), paz mental (Gharibi et al., 2019), estrés (Sinković \& Towler, 2019), fatiga (Linares et al., 2018), rutina e insatisfacción familiar (Trudel et al., 2010).

Flynn y Gow (2015) explicitan una correlación positiva entre las variables psicológicas y la frecuencia de los comportamientos sexuales. A su vez, Robledo et al., (2020) encuentran que quienes reportan nunca sentirse tristes consideraron con mayor importancia su sexualidad en una relación de 7 a 1, de igual manera, sucedió con el reporte de soledad en una relación de 5 a 1. Finalmente, Kleinstäuber (2017) señala que el bienestar psicológico se relaciona significativamente con mantenerse activo sexualmente, satisfacción sexual y adecuado funcionamiento sexual y disminuye la importancia de la sintomatología emocional como un factor significativo.

En cuanto al ámbito relacional, la sexualidad implica vínculos socioemocionales beneficiosos para la SI y CV de los AM (Morell et al., 2018; Ramírez et al., 2017). Una relación afectiva fomenta bienestar integral, genera emociones positivas y placer por vivir (Da Silva et al., 2019). A su vez, tener pareja estable y satisfacción relacional son determinantes de la frecuencia de las conductas sexuales (Gharibi et al., 2019; Jalenques et al., 2020; Perdomo et al., 2013). El estatus civil de casado es decisivo únicamente en mujeres (Yang \& Yan, 2016).

Concerniente al factor cognitivo, se encontraron dos estudios sin resultados concluyentes. Hartmans et al., (2014) concluyen que los AM con mejor funcionamiento cognitivo siguen participando en prácticas sexuales. Por su parte, Hsu et al. (2018) correlacionan el descenso en la función eréctil y menor frecuencia de la actividad sexual con cambios en un screening cognitivo en AM varones. Finalmente, no reportan correlación con la satisfacción sexual reportada.

Por otra parte, la variable autoestima se define como el indicador de aprobación hacia sí mismo (Ramírez et al., 2017) que influye en cómo el AM percibe, siente y experimenta el proceso de envejecimiento sobre su cuerpo (Hurd \& Mahal, 2019). Abarca sentimientos de autoeficacia (Jalenques et al., 2020) y autopercepción del atractivo (Can et al., 2016). La literatura refiere que adecuada autoestima se asocia positivamente con satisfacción sexual (López et al., 2019), comportamientos promotores de salud, vinculación segura con los demás (Uchôa et al., 2016), autocuidado personal (Hernández et al., 2019; Iacub et al., 2020) y mayor frecuencia de conductas 
sexuales (Ramírez et al., 2017; Slack \& Aziz, 2020). En contraposición, su declinación está asociada con infidelidad, insatisfacción sexual (Trudel et al., 2010) y percepción del envejecimiento corporal (Iacub et al., 2020).

De acuerdo con la distribución geográfica, un estudio español indica que el $70 \%$ de los AM declararon tener atractivo sexual y los hombres refirieron sentir menor incomodidad al estar desnudos frente a su pareja (Sinković \& Towler, 2019). Un estudio cubano halla que el 55\% de AM manifestaron baja autoestima (Linares et al., 2018) y otro que el 82\% dijo sentir atracción física por su pareja (Olivet et al., 2014). Asimismo, un estudio canadiense afirma que los hombres están satisfechos con su apariencia y funcionamiento sexual, incluyendo AM con sobrepeso, centrándose en la funcionalidad y desmereciendo la importancia del aspecto físico (Hurd \& Mahal, 2019). Por último, Traeen et al. (2017) identifican que las mujeres AM reportaron menor satisfacción con sus cuerpos y son más vulnerables a presentar alteraciones en su autoestima.

Por lo que se refiere a funcionamiento sexual, el envejecimiento modifica las fases del funcionamiento (Sierra et al., 2014) y respuesta sexual (López et al., 2019). Por su parte, variables físicas que contribuyen a la disfuncionalidad sexual son la debilidad física, menor lubricación (Gharibi et al., 2019) y enfermedades crónicas (Gewirtz et al., 2019; Gharibi et al., 2019; Trudel et al., 2010). La incapacidad de mantener una erección total puede producir ansiedad (Trudel et al., 2010) y pérdida de confianza en sí mismos, puesto que la identidad masculina heterosexual se construye a partir de narrativas referentes al deseo y capacidad sexual (Sinković \& Towler, 2019). En síntesis, déficits en el funcionamiento sexual devienen en pobre CV autoreportada por los AM (Jalenques et al., 2020; Linares et al., 2018).

Desde un orden geográfico, un estudio español evidencia que el $39 \%$ de hombres y el $58 \%$ de mujeres refirieron dificultades en su funcionamiento sexual, aunque, no necesariamente reportaron insatisfacción (Sierra et al., 2014). En Cuba, una investigación determina que el 78\% de AM enunció tener deseo por su pareja (Olivet et al., 2014) y Linares et al. (2018) hallan que el 74\% de mujeres y el $22 \%$ de hombres indicaron falta de motivación hacia comportamientos sexuales. En Brasil, un estudio encuentra que el $29 \%$ de los AM participantes presentaron disfunción sexual y solo la mitad buscó algún tipo de ayuda formal (Uchôa et al., 2016).

Resalta la importancia de la fase del deseo, debido a que predice significativamente la periodicidad de las relaciones sexuales y la conducta masturbatoria de los AM (DeLamater, 2012). Sinković y Towler (2019) aseveran que la disminución considerable o pérdida de deseo repercute en menor frecuencia de la actividad sexual. Pese a la disminución del deseo en la adultez mayor, no desaparece en la mayoría de 
AM (López et al., 2019; Trudel et al., 2014). En otras palabras, Monteagudo et al. (2016) reportan que el $82 \%$ de AM hombres cubanos tuvieron cambios en el deseo pero solo el $4 \%$ refirió nunca experimentarlo.

\subsection{HABILIDADES SOCIOSEXUALES}

Las prácticas sexuales son comportamientos que implican contacto físico y afectivo sin que el objetivo final sea, exclusivamente, la penetración (Olivet et al., 2014). Las más comunes referida por los AM son el coito, caricias, abrazos y masturbación (Flynn \& Gow, 2015; Torres \& Rodríguez, 2019; Yang \& Yan, 2016). Los estudios refieren que del 51\% al 64\% de AM mantienen prácticas sexuales con una periodicidad de 1 a 2 veces por mes (Díaz et al., 2015; Hernández et al., 2019; Olivet et al., 2014; Perdomo et al., 2013; Yang \& Yan, 2016). A su vez la frecuencia y calidad de las conductas sexuales se correlacionan con la CV y SI autodeclarada (Flynn \& Gow, 2015; López et al., 2019; Sierra et al., 2014; Yang \& Yan, 2016).

Concluyendo, Beckman et al. (2014) en su estudio longitudinal a 30 años (1970-2000), evidencian un aumento significativo en la población sueca que mantiene actividad sexual en la adultez mayor, del $12 \%$ al $34 \%$ en mujeres y del $47 \%$ al $66 \%$ en hombres.

Por otro lado, la sexualidad incluye la expresión de deseos, pensamientos y fantasías (Ramírez et al., 2017). Sierra et al. (2014) conceptualizan la asertividad sexual diciendo que "hace referencia a la capacidad para comenzar contactos sexuales, expresar deseos y fantasías, y rechazar actividades sexuales no deseadas" (p.65). Sus determinantes son la fase de cortejo y los juegos previos de seducción que permiten estimular los sentidos a través de abrazos, caricias y besos para potenciar el deseo y la excitación (Gharibi et al., 2019; Iacub et al., 2020).

Esta variable se asocia con el adecuado funcionamiento sexual y mayor frecuencia de las prácticas sexuales en los AM (Traeen et al., 2017), primordialmente, la asertividad de inicio y la ausencia de timidez en hombres españoles (Sierra et al., 2014) y la percepción del control en hombres chinos (Yang \& Yan, 2016). Además, AM con disfunción eréctil reportan mejorías en su $\mathrm{CV}$ al encontrar nuevas formas de intimar satisfactoriamente sin enfocarse en el coito (Sinković \& Towler, 2019). En contraste, mujeres iraníes que no emiten reacciones asertivas frente a comportamientos para iniciar relaciones sexuales reportan menor frecuencia de encuentros sexuales (Gharibi et al., 2019), misma situación ocurre en AM con mandatos sociales fuertemente interiorizados no canalizados asertivamente (López et al., 2019).

Se hallaron ciertos factores que optimizan la sexualidad de los AM. Factores individuales: administrar efectivamente los mandatos religiosos/sociales (Kleinstäuber, 2017; Ménard et al., 
2015) y manejar la ansiedad, vergüenza y duda con la capacidad de tomar riesgos (Ménard et al., 2015). Factores relacionales: percepción de placer mutuo, comunicación en pareja (Cabrera et al., 2018), priorizar la calidad del sexo marital (Kleinstäuber, 2017), apertura a la experiencia, conocimiento, intimidad y niveles emocionales semejantes (Ménard et al., 2015).

Para concluir, sociedades conservadoras posicionan al hombre como quien debería iniciar el contacto sexual lo que afecta, fundamentalmente, la expresión sexual de las mujeres (Yang \& Yan, 2016). A su vez, Ménard et al. (2015) resaltan la importancia de la empatía sexual, entendida como la capacidad de sentir, percibir, atender y responder a las necesidades del otro.

Por otro lado, conocer y entender los cambios fisiológicos, psicológicos y sociales esperables en la adultez mayor permite afrontarlos (Llanes, 2013; Slack \& Aziz, 2020). La información sobre sexualidad se asocia positivamente con asertividad sexual y mayor frecuencia de comportamientos sexuales (Yang \& Yan, 2016), especialmente, mientras más se envejece (DeLamater, 2012). Un estudio polaco asevera que su población tiene un conocimiento moderado en sexualidad (Cybulski et al., 2018). En contraste, estudios latinoamericanos refieren escaso conocimiento de los AM sobre cuestiones sexuales y, mayoritariamente, puntualizaron la necesidad de recibir información al respecto (Díaz et al., 2015; Perdomo et al., 2013; Uchôa et al., 2016). Enfatizando que, una investigación brasileña afirma que el 84\% de AM estudiados no entendieron la distinción entre sexualidad y coito (Uchôa et al., 2016).

Los artículos refieren que los AM buscan aclarar sus dudas con amigos (Cabrera et al., 2018; Da Silva et al., 2019), debido a que no consideran a los especialistas como profesionales preparados para solventarlas y suelen ser la última fuente a la que acuden (Kleinstäuber, 2017; Uchôa et al., 2016). Consecuentemente, el personal sanitario limita su acercamiento y apertura a brindar información sobre el tema (Trudel et al., 2010). Por último, la búsqueda de pornografía, en gran porcentaje de AM, tiene fines educativos (Castro et al., 2018).

La variable transversal a todas las mencionadas es la edad. DeLamater (2012) concluye que más del $72 \%$ de AM exhiben una disminución progresiva en su capacidad sexual conforme envejecen, haciéndose más evidente a partir de los 75 años en ambos sexos (López et al., 2019). Ejemplificando un estudio australiano con una media de 78 años de edad en hombres, declara que el $60 \%$ presentó inactividad sexual, el $46 \%$ obtuvo menor satisfacción sexual y el 70\% refirió descensos en su deseo sexual (Hsu et al., 2018). Además, existe una correlación negativa con el funcionamiento sexual (Sierra et al., 2014; Yang \& Yan, 2016).

En síntesis, la edad disminuye la satisfacción y bienestar sexual a nivel individual, interpersonal y cultural (Traeen et al., 2017). Sin embargo, la sexualidad y sus beneficios pueden mantenerse, ya que no son los años, sino la salud y el manejo de los cambios los que realmente impactan en este ámbito (Gewirtz 
et al., 2019), resaltando que la mayoría de AM son capaces entablar relaciones sexuales y sentir placer en toda la gama de conductas sexuales (Can et al., 2016).

\section{DISCUSIONES Y CONCLUSIONES}

La transición demográfica es la principal justificación para llevar a cabo estas investigaciones siendo conveniente subrayar la carencia de profesionales e información especializada en la temática. En tanto, todas las personas, indistintamente de variables internas y externas, vivenciarán individualmente el proceso de envejecimiento, por lo que es fundamental preguntarse ¿bajo qué condiciones lo hacen?, esencialmente, en un tema vetado como el sexual en esta población estigmatizada.

La sexualidad es un aspecto inherente al ser humano durante toda su existencia, concebida desde diversos factores cognitivos, emocionales, sociales interpersonales, de salud, biológicos, socioeconómicos y culturales, no obstante, escasos artículos reconocen explícita o implícitamente su importancia ni los determina en magnitud ni diferencia por sexos pese a que impactan directamente en el proceso de envejecimiento (Trudel et al., 2010).

Esta revisión sistemática sintetiza la información y facilitará la construcción de nuevos conocimientos teórico-prácticos para desarrollar intervenciones exitosas sociosanitarias que tengan en cuenta la sexualidad, ya que mayoritariamente las vigentes no lo hacen (Jalenques et al., 2020; Traeen et al., 2018; Sinković \& Towler, 2019). Inicialmente, se debe mejorar la representación cultural de los AM para normalizar y legitimar su sexualidad, a través de un proceso educativo, de sensibilización e investigativo con un enfoque comparativo-intercultural que combata las limitaciones sociales, reconociendo sus beneficios y su factor protector desde los postulados del envejecimiento activo.

Las limitaciones encontradas son: 1) Escasas investigaciones sobre sexualidad y sus variables en los AM, principalmente, en poblaciones más envejecidas, con escasos recursos económicos y bajo nivel educativo, destacando que los estudios transculturales han tenido poco éxito replicando resultados (Traeen et al., 2017). 2) Ausencia de un consenso sobre la terminología ni medición de variables. 3) Posiblemente los AM no manejan una definición completa de sexualidad, reduciéndolo al coito, lo que supondría un sesgo relevante al levantar la información. 4) Considerar ampliar el inicio de la adultez mayor a los 70 años atendiendo a la periodización del desarrollo que proponen que los cambios contextuales-históricos modifican la situación social del desarrollo en el ciclo vital (Hsu et al., 2018; Perdomo et al., 2013). 5) Pese a la tentativa de 
comparar los estudios de acuerdo a las regiones los datos obtenidos son insuficientes para evidenciar diferencias. 6) Todo el proceso de revisión lo hizo un único autor.

En conclusión, esta revisión no puede generalizar los resultados encontrados, pero permite la comprensión contextual de las variables psicosexuales y habilidades sociosexuales que determinan la sexualidad de los AM que se instaura como predictora la CV y SI en la adultez mayor. Y se recomienda profundizar el estudio de estas variables en futuras investigaciones. 


\section{REFERENCIAS BIBLIOGRÁFICAS}

Beckman, N., Waern, M., Östling, S., Sundh, V., \& Skoog, I. (2014). Determinants of sexual activity in four birth cohorts of Swedish 70-year-olds examined 1971-2001. Journal of Sexual Medicine, 11(2), 401410. https://doi.org/10.1111/jsm.12381

Cabrera, S., Losa, M. E., Corral, I., Jiménez, R., Rodríguez, R., \& Beccero de Bengoa, R. (2018). Attitudes towards sexuality in elders living alone in a sample of non-institutionalized people. Acta Medica Mediterranea, 34(1), 197-202. https://doi.org/10.19193/0393-6384_2018_1_32

Can, A. R., Sarabia, B., \& Guerrero, J. G. (2016). Factores psicológicos y socioculturales en la vida sexual de los adultos mayores. Revista Iberoamericana de las Ciencias de la Salud, 4(8). https://www.rics.org.mx/index.php/RICS/article/view/29/77

Castro, J., Ballester, R., Giménez, C., \& Gil, B. (2018). Comportamiento sexual online en adultos mayores. International Journal of Developmental and Educational Psychology, 2(2), 89-98. https://doi.org/10.17060/ijodaep.2017.n2.v2.1082

Cybulski, M., Cybulski, L., Krajewska, E., Orzechowska, M., Cwalina, U., \& Jasinski, M. (2018). Sexual quality of life, sexual knowledge, and attitudes of older adults on the example of inhabitants over 60s of Bialystok, Poland. Frontiers in Psychology, 9(1), 1-9. https://doi.org/10.3389/fpsyg.2018.00483

Da Silva, F., Pelzer, M. T., \& Neutzling, B. R. (2019). Attitudes of elderly women regarding the expression of their sexuality. Aquichan, 19(3), 1-12. https://doi.org/10.5294/aqui.2019.19.3.4

Uchôa, Y., Amaral da Costa, D. C., Pamplona, I. A., Saldanha, S. T., Torres, W. M., Chyara, S. (2016). Sexuality through the eyes of the elderly. Rev Bras Geriatr e Gerontol, 19(6), 939-949. https://doi.org/10.1590/1981-22562016019.150189

DeLamater, J. (2012). Sexual expression in later life: A review and synthesis. Journal of Sex Research, 49(2-3), 125-141. https://doi.org/10.1080/00224499.2011.603168

Díaz, H., Lemus, M. N., \& Álvarez, O. (2015). La sexualidad en un grupo de ancianos que asisten a consulta de Urología. Revista de Ciencias Médicas de Pinar Del Río, 19(4), 667-677. http://scielo.sld.cu/pdf/rpr/v19n4/rpr11415.pdf

Ezhova, I., Savidge, L., Bonnett, C., Cassidy, J., Okwuokei, A., \& Dickinson, T. (2020). Barriers to older adults seeking sexual health advice and treatment: A scoping review. International Journal of Nursing Studies, 107(1), 1-16. https://doi.org/10.1016/j.ijnurstu.2020.103566

Fileborn, B., Lyons, A., Hinchliff, S., Brown, G., Heywood, W., Dow, B., Malta, S., \& Minichiello, V. (2017). Improving the sexual lives of older Australians: Perspectives from a qualitative study. Australasian Journal on Ageing, 36(4), 1-7. https://doi.org/10.1111/ajag.12405

Flynn, T. J., \& Gow, A. J. (2015). Examining associations between sexual behaviours and quality of life in older adults. Age and Ageing, 44(5), 823-828. https://doi.org/10.1093/ageing/afv083

Gewirtz, A., Hafford, T., Ayalon, L., Benyamini, Y., Biermann, V., Coffey, A., Jackson, J., Phelan, A., Voß, P., Geiger, M., Zeman, Z. (2019). How do older people discuss their own sexuality? A systematic review of qualitative research studies. Culture, Health and Sexuality, 21(3), 293-308. 
https://doi.org/10.1080/13691058.2018.1465203

Gharibi, T., Gharibi, T., \& Ravanipour, M. (2019). Facilitators and barriers affecting sexual desire in elderly Iranian women: a qualitative study. Sexual and Relationship Therapy, 34(2), 228-241. https://doi.org/10.1080/14681994.2018.1445849

Hartmans, C., Comijs, H., \& Jonker, C. (2014). Cognitive functioning and its influence on sexual behavior in normal aging and dementia. International Journal of Geriatric Psychiatry, 29(5), 441-446. https://doi.org/10.1002/gps.4025

Hernández, M., de la Fuente, S. L., García, N., Hidalgo, A., García, I., \& Cano, M. (2019). Características de la esfera sexual en pacientes adultos mayores. Semergen, 45(1), 37-43. https://doi.org/10.1016/j.semerg.2018.09.007

Heywood, W., Lyons, A., Fileborn, B., Hinchliff, S., Minichiello, V., Malta, S., Barrett, C., \& Dow, B. (2017). Sexual Satisfaction Among Older Australian Heterosexual Men and Women: Findings from the Sex, Age \& Me Study. Journal of Sex and Marital Therapy, 44(3), 295-307. https://doi.org/10.1080/0092623X.2017.1366959

Hsu, B., Hirani, V., Waite, L. M., Naganathan, V., Blyth, F. M., Le Couteur, Seibel, M., Cumming, R., \& Handelsman, D. J. (2018). Temporal associations between sexual function and cognitive function in community-dwelling older men: The concord health and ageing in men project. Age and Ageing, 47(6), 900-904. https://doi.org/10.1093/ageing/afy088

Hurd, L., \& Mahal, R. (2019). “I'm Pleased with my Body”: Older Men's Perceptions and Experiences of their Aging Bodies. Men and Masculinities, 1-17. https://doi.org/10.1177/1097184X19879188

Iacub, R., Hidalgo, P., Winzeler, M. O., Bourlot, V., Gil, M. L., Paz, M., Bellas, M., Machluk, L., Vazquez, R., \& Boggiano, P. (2020). Desarticulando las Fronteras del Erotismo en la Vejez. Research on ageing and social policy, 8(1), 1-24. https://doi.org/10.4471/rasp.2020.4616

Jalenques, I., Rondepierre, F., Rachez, C., Lauron, S., \& Guiguet, C. (2020). Health-related quality of life among community-dwelling people aged 80 years and over: A cross-sectional study in France. Health and Quality of Life Outcomes, 18(126), 1-24. https://doi.org/10.1186/s12955-020-01376-2

Kleinstäuber, M. (2017). Factors associated with sexual health and well being in older adulthood. Current Opinion in Psychiatry, 30(5), 358-368. https://doi.org/10.1097/YCO.0000000000000354

Linares, L. B., Linares, L. P., Hernández, D., \& Lemus, N. M. (2018). Caracterización del comportamiento $\begin{array}{llllll}\text { sexual en } & \text { gn } 3 \text {-35 }\end{array}$ http://www.revgaleno.sld.cu/index.php/ump/article/view/267

Llanes, C. (2013). La sexualidad en el adulto mayor. Revista Cubana de Enfermeria, 29(3), 223-232. https://doi.org/10.18682/pd.v13i0.362

López, H., Medina, M., Hernandez, P., \& Silva, J. M. (2019). Sexualidad en el adulto mayor , conductas y retos - revisión de la literatura. Revista Urol Colomb, 28(1), 121-129. https://doi.org/10.1055/s-00381645849

Ménard, A. D., Kleinplatz, P. J., Rosen, L., Lawless, S., Paradis, N., Campbell, M., \& Huber, J. D. (2015). 
Individual and relational contributors to optimal sexual experiences in older men and women. Sexual and Relationship Therapy, 30(1), 78-93. https://doi.org/10.1080/14681994.2014.931689

Ministerio de Inclusión Económica y Social. (2018). Norma técnica para la implementación y prestación de servicios de atención y cuidado para personas adultas mayores. MIES.

Moher, D., Liberati, A., Tetzlaff, J., Altman, D., \& The PRISMA Group (2009). Preferred Reporting Items for Systematic Reviews and Meta-Analyses: The PRISMA Statement. PLoS Med 6(6). https://doi.org/10.1371/journal.pmed1000097

Monteagudo, G., López, Y., Ledón, L., Gómez, M., Ovies, G., Álvarez Seijas, E., \& Robles, E. (2016). El deseo sexual en varones adultos mayores, su relación con la testosterona sérica y otros factores. Revista Cubana de Endocrinología, 27(1), 29-44. http://scielo.sld.cu/pdf/end/v27n1/end04116.pdf

Morell, V., Ceccato, R., Nebot, J., Chaves, I., \& Gil, M. (2018). Actitudes hacia la sexualidad y bienestar psicológico en personas mayores. International Journal of Developmental and Educational Psychology, 4(1), 77-84. https://doi.org/10.17060/ijodaep.2018.n1.v4.1270

Morris, C., \& Maisto, A. (2009). Desarrollo del ciclo vital. En Morris, C., \& Maisto, A. (13ra. Ed.), Introducción a la Psicología (pp. 381-428). Pearson Education.

Olivet, I., Becerra, Á., \& Ávila, N. (2014). Caracterización de la actividad sexual en adultos mayores del policlínico Gustavo Aldereguía. Revista Electrónica Dr. Zoilo E. Marinello Vidaurreta, 39(5). http://revzoilomarinello.sld.cu/index.php/zmv/article/view/278

Organizacion Mundial de la Salud. (2015). Envejecimiento y ciclo de vida. Organización Mundial de la Salud [citado noviembre 2020]. http://www.who.int/ageing/about/facts/es

Perdomo, I., Oria, N. L., Segredo, A. M., \& Martín, X. (2013). Conducta sexual de los adultos mayores en el área de salud Tamarindo, 2010. Revista Cubana de Medicina General Integral, 29(1), 8-19. http://scielo.sld.cu/pdf/mgi/v29n1/mgi03113.pdf

Ramírez, N., Jiménez, K., \& Guillén, M. (2019). Satisfacción sexual y autoestima en la persona adulta mayor. Sanus, 2(1), 15-24. https://doi.org/10.36789/sanus.vi2.65

Robledo, C., Cardona, D., Cardona, J., Lizcano, D., \& Quintero, Á. (2020). Percepción de la sexualidad en personas mayores de 60 años. Medellín, Colombia. Revista Colombiana de Ciencias Sociales, 11(1), 56-77. https://doi.org/10.21501/22161201.3286

Rubio Aurioles., E. (2015). Lo que todo clínico debe saber de Sexología. Farmacia SA. https://bibliotecaia.ism.edu.ec/Repo-book///Lo-sexologia.pdf

Ševčíková, A., \& Sedláková, T. (2020). The Role of Sexual Activity from the Perspective of Older Adults: A Qualitative Study. Archives of Sexual Behavior, 49(3), 969-981. https://doi.org/10.1007/s10508-01901617-6

Sierra, J., Vallejo, P., Santos, P., Moyano, N., Granados, M., \& Sánchez, M. (2014). Funcionamiento sexual en personas mayores: Influencia de la edad y de factores psicosexuales. Revista Internacional de Andrologia, 12(2), 64-70. https://doi.org/10.1016/j.androl.2013.10.002 
Sinković, M., \& Towler, L. (2019). Sexual Aging: A Systematic Review of Qualitative Research on the Sexuality and Sexual Health of Older Adults. Qualitative Health Research, 29(9), 1239-1254. https://doi.org/10.1177/1049732318819834

Slack, P., \& Aziz, V. (2020). Sexuality and sexual dysfunctions in older people: a forgotten problem. BJPsych Advances, 26(3), 173-182. https://doi.org/10.1192/bja.2019.80

Srinivasan, S., Glover, J., Tampi, R. R., Tampi, D. J., \& Sewell, D. (2019). Sexuality and the Older Adult. Current Psychiatry Reports, 21(97), 1-9. https://doi.org/10.1007/s11920-019-1090-4

Torres, S., \& Rodríguez, B. (2019). Percepciones de la sexualidad en personas mayores: una revisión sistemática de estudios cualitativos. Rev Esp Salud Pública, 93(4), 1-17. http://scielo.isciii.es/pdf/resp/v93/1135-5727-resp-93-e201909059.pdf

Traeen, B., Carvalheira, A., Hald, G., Lange, T., \& Kvalem, I. (2018). Attitudes Towards Sexuality in Older Men and Women Across Europe: Similarities, Differences, and Associations with Their Sex Lives. Sexuality and Culture, 23(1), 1-25. https://doi.org/10.1007/s12119-018-9564-9

Traeen, B., Carvalheira, A., Kvalem, I., Štulhofer, A., Janssen, E., Graham, C., Hald, G. \& Enzlin, P. (2017). Sexuality in Older Adults (65+) —An Overview of The Recent Literature, Part 2: Body Image and Sexual Satisfaction. International Journal of Sexual Health, 29(1), 11-21. https://doi.org/10.1080/19317611.2016.1227012

Trudel, G, Turgeon, L., \& Piché, L. (2010). Marital and sexual aspects of old age. Sexual and Relationship Therapy, 25(3), 316-341. https://doi.org/10.1080/14681991003750467

Trudel, G., Dargis, L., Villeneuve, L., Cadieux, J., Boyer, R., \& Préville, M. (2014). Marital, sexual and psychological functioning of older couples living at home: The results of a national survey using longitudinal methodology (Part II). Sexologies, 23(2), 35-48. https://doi.org/10.1016/j.sexol.2013.03.007

Yang, S., \& Yan, E. (2016). Demographic and psychosocial correlates of sexual activity in older Chinese people. Journal of Clinical Nursing, 25(5-6), 672-681. https://doi.org/10.1111/jocn.12998 